\title{
Lithium-Beryllium-Boron and Oxygen in the Early Galaxy
}

\author{
Elisabeth Vangioni-Flam \\ Institut d'Astrophysique de Paris, CNRS, 98 bis Boulevard Arago, \\ 75014, Paris, France \\ Michel Cassé \\ Service d'Astrophysique, DAPNIA, CEA Orme des Merisiers, 91191 Gif \\ sur Yvette, CEDEX, France \\ and Institut d'Astrophysique de Paris, CNRS, 98 bis Boulevard Arago, \\ 75014, Paris, France
}

\begin{abstract}
Oxygen is a much better evolutionary index than iron to follow the history of Lithium-Beryllium-Boron ( $\mathrm{LiBeB}$ ) since it is the main producer of these light elements at least in the early Galaxy. The $\mathrm{O}-\mathrm{Fe}$ relation is crucial to the determination of the exact physical process responsible for the $\mathrm{LiBeB}$ production. Calculated nucleosynthetic yields of massive stars, estimates of the energy cost of Be production, and above all recent observations reported in this meeting seem to favor a mechanism in which fast nuclei enriched in $\mathrm{He}, \mathrm{C}$ and $\mathrm{O}$ arising from supernovae are accelerated in superbubbles and fragment on $\mathrm{H}$ and $\mathrm{He}$ in the interstellar medium.
\end{abstract}

\section{Introduction}

Lithium-Beryllium-Boron take a special status in the general framework of nucleosynthesis. These nuclei are indeed of exceptional fragility and they are destroyed in stars above about 1 million degrees. The main formation process available is spallation, i.e. fragmentation of medium light isotopes (CNO) leading to lighter species such as ${ }^{6} \mathrm{Li},{ }^{7} \mathrm{Li},{ }^{9} \mathrm{Be},{ }^{10} \mathrm{~B}$ and ${ }^{11} \mathrm{~B}$. The physical parameters of the spallation mechanism are fourfold: i) the production cross sections as a function of energy are fairly well measured in the laboratory; ii) the source composition of the energetic component; iii) the associated (injection) energy spectrum; iv) the target composition. In the following, we describe the two reverse spallative processes able to produce $\mathrm{LiBeB}$ and we confront them to observational constraints. We show how the relation between oxygen and iron in the halo phase is determining to discriminate between the two processes.

\section{Spallation processes and astrophysical sites}

The pioneering article of Meneguzzi, Audouze and Reeves (1971) offered the first quantitative explanation of the local abundances of $\mathrm{LiBeB}$, at a time when only 
cumulated abundances in the Solar System were available. The standard Galactic Cosmic Rays (GCR), essentially composed of fast protons and alphas collide with $\mathrm{CNO}$ nuclei sitting in the interstellar medium to yield measured $\mathrm{LiBeB}$ abundances. But the observed isotopic ratios of $\mathrm{Li}$ and $\mathrm{B}$ were not reproduced. A stellar source of ${ }^{7} \mathrm{Li}$ was made necessary. For ${ }^{11} \mathrm{~B}$ a complementary ad hoc spallative source of low energy was invoked.

Introducing the time parameter, i.e. taking into account the fact that the amount of CNO varies in the ISM as well as the flux of cosmic rays (protons and alphas), presumably like the rate of supernovae, itself responsible for the increment of metallicity, leads to the following evolution: the abundance of a given light element increases like the square of the CNO abundance (or as a good approximation to O). Thus according to the classical tradition in Galactic evolution, the production of $\mathrm{LiBeB}$ by the GCR is called "secondary".

In the nineties, measurements of $\mathrm{Be} / \mathrm{H}$ and $\mathrm{B} / \mathrm{H}$ from KECK and HST, together with $[\mathrm{Fe} / \mathrm{H}]$ in low metallicity halo stars came to set strong constraints on the origin and evolution of $\mathrm{LiBeB}$ isotopes. The evolution of $\mathrm{BeB}$ was suddenly uncovered over about $10 \mathrm{Gyr}$, taking $[\mathrm{Fe} / \mathrm{H}]$ as an evolutionary index. The linearity between $\mathrm{Be}, \mathrm{B}$ and iron came as a surprise since a quadratic relation was expected from the standard GCR mechanism. It was a strong indication that the standard GCRs are not the main producers of $\mathrm{LiBeB}$ in the early Galaxy. A new mechanism of primary nature (production rate independent of the interstellar metallicity, i.e. $\mathrm{BeB}$ abundances $\propto 0$ ) was required to reproduce these observations: it has been proposed that low energy $\mathrm{CO}$ nuclei (a few tens $\mathrm{MeV} / \mathbf{n}$ ) produced and accelerated by massive stars (WR and SN II in superbubbles, i.e. cavities in the interstellar medium excavated by the winds and explosion of massive stars) fragment on $\mathrm{H}$ and $\mathrm{He}$ at rest in the ISM. This low energy component (LEC) has the advantage of coproducing Be and B in good agreement with the ratio observed in Pop II stars (Vangioni-Flam et al. 2000; Ramaty et al. 2000).

The term "metallicity" up to now has been ambiguously defined. In fact, all the above argumentation assumes that $\mathrm{CNO} / \mathrm{Fe}$ is constant at $[\mathrm{Fe} / \mathrm{H}]$ less than -1 . Since oxygen is the main progenitor of $\mathrm{BeB}$, the apparent linear relation between $\mathrm{BeB}$ and $\mathrm{Fe}$ could be misleading if $\mathrm{O}$ was not strictly proportional to $\mathrm{Fe}$. Thus the pure primary origin of $\mathrm{BeB}$ in the early Galaxy could be questioned.

If $\mathrm{O} / \mathrm{Fe}$ is constant, the GCR process is insignificant due to the paucity of the ISM in CNO in the halo phase. The LEC process is obviously predominant, since it is free from the ISM metallicity, relying on freshly synthesized $\mathrm{He}, \mathrm{C}$, and O. Progressively, following the general enrichment of the ISM, GCR gain importance. Neutrino spallation plays its marginal role, increasing the abundance of ${ }^{11} \mathrm{~B}$.

Recent observations by Israelian et al. (1998) and Boesgaard et al. (1999) (IB) showing a neat slope in the O-Fe plot have seeded a trouble. If they are verified, the whole interpretation has to be modified, giving a larger role to the secondary process in the halo phase (Fields \& Olive 1999). Nevertheless, the primary component is also required in the very early Galaxy. However, these observations are considered as controversial, and the whole session is centered on this point. What is the right $\mathrm{O}-\mathrm{Fe}$ correlation? This point is crucial to translate the $\mathrm{Be}, \mathrm{B}-\mathrm{Fe}$ relations into $\mathrm{B}, \mathrm{Be}-\mathrm{O}$ ones. In the $\mathrm{IB}$ observations, $[\mathrm{O} / \mathrm{Fe}]=-0.35$ $[\mathrm{Fe} / \mathrm{H}]$, and consequently $\log (\mathrm{Be} / \mathrm{H})$ is proportional to $1.55[\mathrm{O} / \mathrm{H}]$. Fields \& 
Olive (1999) use this relation to rehabilitate the classical standard GCR as the progenitor of $\mathrm{LiBeB}$. But, beyond the observational questioning, this scenario meets with theoretical difficulties. The energy cost to produce a single Be nucleus is unfavorable but not prohibitive since this cost is plagued by large uncertainties (Fields et al. 2000). The main difficulty is that the stellar supernova yields integrated in a Galactic evolutionary model cannot fit the new $\mathrm{O} / \mathrm{Fe}$ data (F. Matteucci, this meeting). Moreover, the observational $[\alpha / \mathrm{Fe}]$ vs $[\mathrm{Fe} / \mathrm{H}]$, where $\alpha$ $=\mathrm{Mg}, \mathrm{Si}, \mathrm{Ca}, \mathrm{Ti}$, show a plateau from $[\mathrm{Fe} / \mathrm{H}]=-4$ to -1 . It would be surprising that oxygen does not follow the $\mathrm{Mg}, \mathrm{Si}$ and $\mathrm{Ca}$ trends since all these nuclei are produced by the same massive stars. If the trend expressed in this meeting, namely that $[\mathrm{O} / \mathrm{Fe}]$ is approximatively constant or slightly varying in the halo phase is confirmed, then the theoretical situation is clarified and the primary component made necessary in the early Galaxy, until at least $[\mathrm{Fe} / \mathrm{H}]=-1$.

\section{Conclusion}

The synthesis of $\mathrm{LiBeB}$ in the halo proceeds through nuclear spallation, essentially by the break up of oxygen and the $\alpha+\alpha$ reaction. The observed relation between $\mathrm{BeB}$ and $\mathrm{Fe}$ has to be translated through the $\mathrm{O}-\mathrm{Fe}$ relation into a $\mathrm{BeB}-$ $O$ which is representative of the relevant physical production process. And then the observational $\mathrm{O}-\mathrm{Fe}$ relation is determining, except if $\mathrm{Be}, \mathrm{B}$ and $\mathrm{O}$ are measured simultaneoulsy in stars (A.M. Boesgaard and K. Cunha, this meeting). Anyway the primary component is required in the halo phase, then afterwards the secondary process takes over; the question is therefore at which metallicity the transition occurs, the answer depending on the behaviour of $[\mathrm{O} / \mathrm{Fe}]$. The ideal test of the scenarios would be to get the evaluation of $\mathrm{O}, \mathrm{Fe}, \mathrm{Mg}, \mathrm{Be}, \mathrm{B}$, $\mathrm{Li}$ abundances in the same stars. Impressive progress is awaited from the VLT. Gamma ray line astronomy, through the european INTEGRAL satellite, to be launched in 2002, will help to constrain the energy spectrum and intensity of LEC.

\section{References}

Boesgaard, A.M., King, J.R., Deliyannis, C.P. \& Vogt, S.S. 1999, AJ, 117, 492 Fields, B.D. \& Olive, K.A. 1999, ApJ, 516, 797

Fields, B.D., Olive, K.A., Cassé, M. \& Vangioni-Flam, E. 2000, A\&A, submitted Israelian, G., García López, R.F. \& Rebolo, R. 1998, ApJ, 507, 357

Meneguzzi, M., Audouze, J. \& Reeves, H. 1971, A\&A, 15, 337

Ramaty, R., Scully, S.T., Lingenfelter, R.E. \& Kozlovsky, B. 2000, ApJ, 534, 747

Vangioni-Flam, E., Cassé, M. \& Audouze J. 2000, Phys. Rep., 333-334, 365 